\title{
L'anxiété de la communication interculturelle dans le cas d'urgence et son élimination
}

\section{Shan Bo}

\section{Q OpenEdition}

1 Journals

Édition électronique

URL : http://journals.openedition.org/communicationorganisation/3431

DOI : 10.4000/communicationorganisation.3431

ISSN : 1775-3546

Éditeur

Presses universitaires de Bordeaux

\section{Édition imprimée}

Date de publication : 1 juin 2006

Pagination : 262-269

ISSN : 1168-5549

\section{Référence électronique}

Shan Bo, "L'anxiété de la communication interculturelle dans le cas d'urgence et son élimination », Communication et organisation [En ligne], 29 | 2006, mis en ligne le 21 juin 2012, consulté le 19 décembre 2020. URL : http://journals.openedition.org/communicationorganisation/3431 ; DOI : https://doi.org/10.4000/communicationorganisation.3431

Ce document a été généré automatiquement le 19 décembre 2020.

(c) Presses universitaires de Bordeaux 


\title{
L'anxiété de la communication interculturelle dans le cas d'urgence et son élimination
}

\author{
Shan Bo
}

1 La communication interculturelle présuppose un environnement social et psychologique de l'égalité, de la paix et de la sécurité. Dans un tel environnement, la possibilité de comprendre les autres cultures augmente et on peut ainsi communiquer librement dans le monde multiculturel. Mais c'est là, la communication interculturelle idéale. La communication interculturelle dans la réalité est toujours effectivement déséquilibrée. Ces déséquilibres se manifestent par les luttes pour le pouvoir quand surtout les deux mondes culturels sont conflictuels et exclusifs. La communication interculturelle entrant en état d'urgence produit de l'anxiété culturelle et sociale, laquelle affecte par extension l'échange et la communication entre cultures.

2 L'état d'urgence se réfère d'une part à la situation d'imminence avec la pression, le danger et le risque extérieurs, d'autre part à l'état psychologique d'urgence intérieure. L'état d'urgence de vie se montre par l'état d'urgence culturelle, comme la terreur se révèle sous la forme de la haine et du préjugé de la xénophobie. L'expansion de l'hégémonie culturelle a causé la disparition de la diversité culturelle. On a perdu le droit à la créativité et on craint de devenir des hommes marginalisés. En l'occurrence, soit on se détourne de la communication culturelle normale - probablement pris dans le piège de la fierté et du préjugé irrationnel - et de l'équilibre de Nash dans le dilemme $\mathrm{du}$ prisonnier en raison de la non-coopération rationnelle parmi les joueurs, soit deviendra anxieux dans la communication interculturelle Cette anxiété sera une entrave à l'objectif de la communication interculturelle. Il me semble que, de plus en plus, nous nous ressentons anxiété et précarité. En même temps nous nous trouvons entourés par l'état d'urgence sous des formes diverses. Ceci souligne les conséquences de la globalisation, l'arrivée de l'ère de l'information, le monde du rétréci, la croissante vitesse du développement, l'accroissement du décalage entre les riches et les pauvres, les aggravantes crises économiques et sociales et les recours fréquents au terrorisme. 
Généralement, l'anxiété est une description de cette expérience émotionnelle de la tension, la peur et la crainte. L'anxiété apparaît quand on est confronté au danger, au péril ou à la menace, quand on s'attend à un bon résultat et qu'on reçoit l'échec et la perte d'intérêts importants ou de quelque chose qu'on apprécie. L'anxiété nous révèle à nous-mêmes, et enfin nous pouvons reconnaitre et reprendre les choses que nous apprécions. Nous chercherons peut-être à établir une nouvelle échelle de valeurs. Cependant, en même temps, l'anxiété nous entraîne vers une échelle de valeurs trop absolue et individuelle. Nous perdrons donc là d'autres références culturelles. L'élimination des anxiétés que j'indique est l'élimination de leurs effets négatifs. On ne peut pas éliminer l'anxiété elle-même parce qu'elle fait partie de la vie. Nous sommes face à l'anxiété. Elle nous montre à quelle menace nous sommes confrontés, et si nous avons les moyens d'y répondre. L'anxiété de la communication interculturelle se manifeste par l'anxiété de l'identité culturelle. L'identité culturelle est la position culturelle reconnue par soi, autrui et la communauté. L'anxiété de l'identité culturelle est principalement le résultat de la relation déséquilibrée entre l'environnement culturel et les individus. On a le désir 'appartenance. La confirmation de l'identité est la nécessité inhérente et inconsciente du comportement. Les individus obtiennent la sécurité psychologique et persistent à entretenir, protéger et renforcer le sens de la sécurité, quand notamment ils doivent changer de mode de vie, par exemple en raison de l'immigration. Des questions comme « qui suis-je? ", « Pouvons nous nous identifier aux nouvelles communautés culturelles?», "Est-ce que je dois abandonner mon identité ancienne et me joindre à la nouvelle communauté culturelle?", apparaissent quand on ne peut pas recevoir une explication satisfaisante et complète. Lorsqu'on éprouve de la difficulté à équilibrer son monde interne et à rétablir la relation entre l'environnement culturel et soi, on est rempli par l'anxiété, laquelle possiblement causera l'instabilité sociale et engagera le peuple dans le vide individuel.

La constitution de l'identité culturelle comporte trois parties: la manifestation, la structure psychologique et l'image culturelle. La manifestation est l'expression de l'identité culturelle, comme, par exemple, les mets qu'on mange, les langues qu'on parle, les livres qu'on lit, les amis qu'on se fait. De telles choses constituent les éléments nécessaires de l'identité, mais n'en sont pas les éléments essentiels. La structurepsychologique se rapporte à l'identité culturelle. L'image culturelle, c'est la manière dont les autres, les étrangers, apprécient la sélection, l'interprétation et l'évaluation de l'entité.

5 Dans l'environnement social et psychologique de l'égalité, de la paix et de la sécurité, les conflits extérieurs et les tensions intérieures ne se forment pas parmi les éléments de l'identité, mais les éléments de l'identité trouvent leurs positions respectives, s'attirent, et enfin forment un équilibre. Donc l'identité individuelle et interculturelle sont relatives dans le monde globalisé. Pourtant, les conflits et les rejets détruisent toujours l'équilibre et forment une tension culturelle, comme le moment de la privation du discours comme lorsqueles Allemands ont envahi la France. Ceci est décrit dans La Dernière Classe par Alphonse Daudet Ou lorsque le premier ministre japonais Junichiro Koizumi en visitant le Temple Yasukuni a déclenché un affrontement culturel et psychologique. En ce moment, des peuples entrent dans l'identification culturelle uniforme d'une communauté font naîtreun affrontement farouche entre "nous » et «ils», croyant pieusement dans les valeurs de leur propre communauté et en nous laissant présupposer que ces valeurs pourraient être distordues et blessées. En Chine, 
des communautés nationales sont préoccupées par l'aphasie culturelle que peut amener la pénétration culturelle occidentale uniforme. Les peuples frontaliers qui transcendent la frontière culturelle sont probablement menacés par la crise culturelle de l'identité dont parle A. Giddens. La crise est généralement accompagnée par l'anxiété psychologique et le dépaysement des sens. Ils ne savent plus qui ils sont et perdent la direction, perte qui génère les troubles forts de l'instabilité et du dérangement interne qui définissent l'anxiété de l'identification culturelle. S. Buck Pearl, qui a vécu l'époque où la culture chinoise a rencontré la culture américaine, dit : "J'ai grandi dans un monde double - le monde petit et prospère de ses parents américains et le monde beau et franc de la Chine - il y a un mur entre eux. Dans le monde chinois, je dis, je fais, je mange comme le peuple chinois. Nous pouvons échanger les émotions. Quand je suis dans le monde américain, je ferme la porte à un monde autre. » Entre l'identité chinoise et l'américaine, un flottement existait. Lorsque les deux mondes furent en conflit et se rejetèrent, elle ressentit qu'elle était en train de s'exclure des deux mondes. Dans son for intérieur, elle ne pouvait pas posséder un monde distinct. Elle vivait un véritable exil.

6 L'anxiété de l'identification culturelle peut s'expliquer par les théories de «l'indifférence de soi », «l'attribution» et "l'incoordination de la cognition». La théorie de "l'différence de soi" de E. T. Higgins explique que nous sommes différents de l'homme que nous espérons être, ceci est l'origine de l'anxiété. L'avis qu'on a de soi est le concept de soi, et l'homme qu'on voudrait être s'appelle l'orientation de soi. E. T. Higgins explique que l'orientation de soi transcende toujours le concept de soi. Les petites différences entre eux servent d'encouragement pour l'individu. Mais, quand les différences sont si grandes et que l'individu a conscience de ces différences au point de ressentir de la gêne émotionnelle, apparaissent le dérangement et la frustration spirituelle; donc l'anxiété.

7 Dans la communication interculturelle, il y a plusieurs possibles. Les deux principales sont : une orientation de soi de "la découverte de la racine culturelle", dans laquelle on croit qu'on appartient à une culture pure qui indique l'identification nationale, historique et culturelle de l'homme, comme la culture chinoise, la culture indienne, la culture de la Égypte, la culture du genre ou la culture de classe. L'autre orientation de soi est une identification à des cultures diverses qui s'opposent et on ne peut pas céder soi au pouvoir culturel hors de celui de soi. Nous devons participer aux différents jeux culturels et sociaux des mots, nous engager dans la compliquée structure culturelle consciemment, comme les peuples de la frontière qui sont entre deux cultures. Quand la communication culturelle est en état de tension ou de conflit et de rejet culturel, on trouve les différences entre la situation réelle et l'orientation de soi. Plus il y a conscience de soi plus grande est la fluctuation, en conséquence de quoi l'alerte est élevée et l'obsession et l'anxiété fortes. Pour les peuples qui ont une orientation de soi de type "découverte de la racine culturelle", la conscience des différences se manifeste par le détour des caractères de la culture de soi sous la pression culturelle. Donc, on change peut-être l'image culturelle de soi, on abandonne les droits culturels de soi. Nous courons alors le risque du développement de la communauté culturelle. Pour les peuples avec une identification à des cultures diverses, la conscience des différences se manifeste par l'exacerbation du conflit culturel, la perte de l' existence culturelle, l'abandon au pouvoir extérieur, sélection invariable de la culture ou la dépendance par rapport à une culture du superordinateur. Normalement la conscience des différences se manifeste dans une situation spécifique. En cas d'avènement du 
conflit et du danger culturel, l'individu comparera automatiquement les comportements culturels et les sélections culturelles avec l'orientation de soi. Quand il trouve que les différences dégradent sa dignité, il ressentira l'anxiété. Parce que la conscience se concentre sur les différences, elle causera la confusion émotionnelle et la dévaluation de soi. Ainsi, on évitera l'évaluation de soi, laquelle est impossible. En ce cas, on s'avilira pour protéger sa dignité, éviter ses problèmes, et on justifiera la frustration culturelle. Mais cette dévaluation de soi qui causera la perte de la valeur culturelle est différente de la critique culturelle de soi, laquelle peut retrouver l'origine de la valeur pour développement culturel de soi avec la confiance et le rationalisme. L'anxiété s'exacerbe, alors.

8 La dévaluation culturelle de soi relève du problème de l'attribution. L'attribution se réfère aux causes des comportements. On trouve la cause du comportement parmi divers facteurs possibles. L'attribution relève plus des facteurs émotionnels et de la psychologie. Les résultats de l'attribution affectent les émotions, comportements et attitudes de l'homme. L'attribution est une activité de la subjectivité. Par rapport à l'attribution, le facteur qui affecte les émotions de l'homme n'est souvent pas le résultat, mais la perception de la cause. Normalement, l'expérience émotionnelle des résultats est transitoire. Même les expériences négatives ne peuvent pas engager l'individu à l'anxiété. Les expériences émotionnelles sont à l'origine des évaluations objectives des comportements. En ce moment, on ne peut pas se satisfaire de la perception des résultats, il faut trouver la cause des résultats. Quand on pense à la cause des comportements, les moyens différents et les expériences émotionnelles différentes apparaissent. Dans la plupart des situations, les résultats ne sont pas importants. Ce sont les causes qui sont importantes. Dans la communication culturelle, les résultats de l'attribution sont compliqués et variable. Pour clarifier les valeurs de la communauté culturelle à laquelle on appartient, il faut réfléchir au comportement, et trouver la cause interne du changement culturel négatif dans les crises, ainsi on peut trouve la cause réelle de la frustration culturelle, laquelle est la manifestation de la confiance culturelle et démontre un esprit culturel de nature à prendre la décision de soi. Si on a l'attitude envers les valeurs de la communauté culturelle à laquelle on appartient, et qu'on évalue les valeurs de la communauté culturelle à laquelle on appartient par rapport aux valeurs de la communauté culturelle des autres, on dégradera et blessera sa dignité culturelle, laquelle causera des sentiments négatifs d'infériorité et de renoncement, et même l'anxiété quant à l'identification culturelle de soi. Si on attribue la crise culturelle au facteur politique, économique ou religieux, on croit seulement à la fatalité. Les expectatives du développement de la communauté culturelle à laquelle on appartient diminuent, causant les expériences émotionnelles de l'infériorité, le désappointement et le vide.

9 Dans le processus de recherche de la cause de la crise culturelle, l'incongruité cognitive suscite l'anxiété culturelle. Les théories concernant la cognition exposent que généralement on incline à tenir la consistance de la cognition et à rationaliser les incongruités extérieures de la consistance cognitive ou psychologique. Avec l'arrivée de nouveaux facteurs cognitifs et l'incongruité cognitive entre l'individu et les autres peuples dans la communauté culturelle, l'incongruité cognitive de l'individu apparait, laquelle normalement cause l'incongruité logique ou de l'attitude, du comportement, avec la conséquence de l'état de l'anxiété. Par exemple, quand nous, Chinois nous évoquons l'histoire de l'oppression par l'impérialisme, ou quand nous étions confrontés aux événements de la collusion d'un avion américain et d'un avion chinois, quand eut 
lieu le bombardement de l'ambassade chinoise en Yougoslavie, nous répétons deux facteurs contradictoires : " les arriérés seront les battus ", "suivre le chemin de la paix et du développement ». Le premier signifie que dans un monde où les forts tyrannisent les faibles, la culture des faibles est inévitablement battue par celle des forts. Ainsi, il faut que les faibles s'agrandissent, rejoignent le cruel système mondial de la compétition : la survie est un enjeu de dynamisme. La signification du second est que la Chine a besoin d'un environnement de paix et de développement. La contradiction est : comment pouvons-nous avoir la paix pendant que nous reconnaissons le principe de la survie du plus tonique? Si nous suivons le chemin de la paix et du développement, comment pouvons-nous nous confronter aux challenges de la compétition. L'anxiété du Chinois réside dans sa contradiction. L'histoire et la réalité nous ont dit que si le décalage dans la communication interculturelle existe, on ne peut pas éliminer les conflits et le rejet culturel. Si les nécessités de la sécurité sont dans les menaces, l'anxiété de notre identification culturelle ne s'arrête pas. L'identification culturelle incarne la dépendance spirituelle du peuple, comme "la clé des prisons de soi.» Quand nous en avons besoin, nous nous cachons en nous-même, ce qui montre la contradiction de l'homme. D'une part, nous trouvons le préjugé, le biais et la discrimination, d'autre part, nous marquons le territoire culturel de soi, maintenant la supériorité de la culture de soi et acceptant le préjugé, le biais et la discrimination nouvelle. Donc, les cultures sons toujours dans les conflits et les rejets. Les nécessités de la sécurité ne sont pas satisfaites.

Dans la communication interculturelle quotidienne, nous trouvons les différences culturelles. Les communications et les dialogues parmi les peuples des différentes communautés culturelles manquent. Nous sommes sensibles aux relations du pouvoir, à la domination de la culture d'autrui. Et en l'état d'urgence, l'anxiété dans la communication interculturelle devient presque un désespoir: c'est impossible de communiquer parmi les peuples de différentes cultures. Alors nous demandons de l'aide aux solidarités universelles. Nous montrons notre souci pour autrui. Mais, ce discours culturel éthique deviendra un discours culturel politique, une relation de contrôle entre les dominants et les exclus. Dans les reportages sur le Tsunami de l'Asie du Sud-Est, nous trouvons dans les médias d'une part que la sympathie de l'homme diminue la distance entre les gens sous la menace des catastrophes naturelles, d'autre part des problème apparaissent quand on offre sa aide : Est-ce que l'aide des Américains fait partie de leur stratégie globale ? L'aide des Chinois relève-t-elle d'une ambition de la Chine à dominer l'Asie. Evidemment, on met son espoir dans l'entente mutuelle, mais on a ses intérêts respectifs, et la communication culturelle se joue ainsi. Pour se débarrasser de la mauvaise situation, il faut qu'on change l'opinion de la communication culturelle. John Durham Peters, spécialiste américain en communication, indique, après les examens des dilemmes dans les communications de l'homme et les théories paradoxales, que les défis de la communication ne sont pas dans l'adhésion à la culture de soi, mais dans la tolérance à l'égard d'autrui. L'autre ne peut pas nous regarder comme nous-mêmes parce que nous sommes différents. Nous ne pouvons pas vivre dans l'isolement. Ainsi, notre problème n'est pas de pouvoir communiquer. Notre problème est vraiment de pouvoir s'aimer et se tolérer. Nous ne pouvons pas regarder l'autre avec la perspective de nous-mêmes. Il faut que nous tolérions l'autre et que nous reconnaissions ses droits à l'existence et à l'appartenance à un système culturel autre. Effectivement toutes les anxiétés vraies sont relatives à l'autre. Donc il nous faut regarder la communication culturelle dans la perspective de 
l'autre. Si nous regardons la communication culturelle dans la perspective de l'autre, nous pouvons soulager la tension. Nous regardons la culture de points de vue divers et protégeons les droits de chacun.

11 La cause de l'anxiété de l'identification culturelle est dans le trop d'attention que nous portons à l'entité culturelle. L'entité culturelle assure les droits des entités, mais ne peut pas garantir les responsabilités. On entre peut-être dans le non-équilibre des droits et responsabilités. L'entité culturelle contribue à la manifestation des valeurs culturelles de soi, laquelle se montre unilatérale de la culture et expulse les valeurs d'autrui. Pour se débarrasser de l'anxiété, il faut construire l'identification culturelle à l'aide du concept de "l'inter entité». S. Hall, spécialiste américain de la culture disait: « on ne peut pas considérer l'identification finie et réalisée par les nouvelles pratiques sociales et culturelles. Il faut que nous la considérions comme une production éternelle et la manifestation de la construction intérieure, et non pas extérieure. » Ainsi, on peut abandonner la fausse identification intégrale, laquelle est mise au centre du contrôle, et revenir donc à la relation de l'homme. Ce qui nous fait transcender les barrières du centralisme culturel en le mettant en dépendance avec la force de l'éthique.

\section{AUTEUR}

\section{SHAN BO}

Institut du Journalisme et de la Communication - Université de Wuhan 